\title{
Implementación de una actividad física en residentes de pediatría y síndrome de Burnout
}

\section{Implementation of a physical activity in pediatric residents and Burnout síndrome}

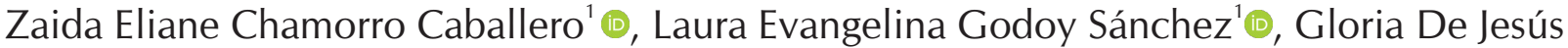 \\ Martínez', Mirta N Mesquita Ramirez'을
}

${ }^{1}$ Hospital General pediátrico Niños de Acosta Nu. San Lorenzo, Paraguay.

\section{RESUMEN}

Introducción: La residencia es un periodo en la formación del médico muy diferente a lo aprendido en la universidad. Objetivo: evaluar el efecto de la actividad física en residentes de pediatría, sobre el Síndrome de Burnout. Materiales y Métodos: Estudio de intervención no aleatorizado controlado. Los residentes de Pediatría fueron asignados a recibir clases de Zumba 2 horas semanales por 10 semanas (grupo estudio) o ninguna intervención (grupo control). Se midió el Síndrome de Burnout utilizando el cuestionario Maslach Burnout Inventory pre y post intervención en ambos grupos. Otras variables estudiadas fueron: edad, género, actividad física y social, estructura familiar y años de residencia. Los datos fueron analizados con SPSS. Se consideró un error alfa inferior al 5\%. El protocolo fue aprobado por el comité de ética del hospital, con consentimiento de los participantes. Resultados: Participaron 47 residentes, 23 en el grupo estudio y 24 en el grupo control. El Síndrome de Burnout pre- intervención fue 52,2 \% y 37,5\% en el grupo estudio y el grupo control respectivamente $p=0,31$. Después de la intervención fue de $17,4 \%$ y 45,8\% en el grupo estudio y control respectivamente. OR: 0,25 (IC95\% 0,65-0,9) p=0,03. El beneficio absoluto fue del $35 \%$ y el número necesario para tratar 2,8. Conclusiones: El ejercicio físico 2 horas semanales mitigó el síndrome de burnout en residentes de Pediatría, con un beneficio absoluto del $35 \%$.

Palabras claves: Burnout, agotamiento psicológico, Pediatría, actividad física.

\section{ABSTRACT}

Introduction: Residency is a period in the training of the doctor very different from what was learned at the university level. Objective: evaluate the effect of physical activity in pediatric residents regarding Burnout Syndrome. Materials and Methods: This was a nonrandomized controlled intervention study. Pediatric residents were assigned to receive Zumba classes 2 hours per week for 10 weeks (study group) or no intervention (control group). Burnout Syndrome was measured using the Maslach Burnout Inventory questionnaire pre and post intervention in both groups. Other variables studied were: age, gender, physical and social activity, family structure and years of residence. The data were analyzed with SPSS. An alpha error of less than 5\% was considered. The protocol was approved by the ethics committee of the hospital, with participants' consent. Results: 47 residents participated, 23 in the study group and 24 in the control group. Pre-intervention Burnout Syndrome was 52.2\% and $37.5 \%$ in the study group and the control group, respectively, $p=0.31$. After the intervention it was $17.4 \%$ and $45.8 \%$ in the study and control group respectively. OR: 0.25 (95\% CI $0.65-0.9) \mathrm{p}=0.03$. The absolute benefit was $35 \%$ and the number needed to treat 2.8. Conclusions: Physical exercise for 2 hours a week alleviated burnout syndrome in Pediatric residents, with an absolute benefit of $35 \%$.

Keywords: Burnout, psychological exhaustion, Pediatrics, physical activity.

Correspondencia: Laura Evangelina Godoy Sánchez Correo: legodoys@gmail.com

Conflicto de interés: Los autores declaran no poseer conflicto de interés

Recibido: 05/01/2021 Aceptado:12/02/2021

DOI: https://doi.org/10.31698/ped.48012021006

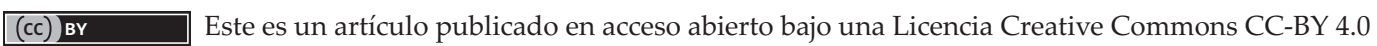




\section{INTRODUCCIÓN}

La residencia es un periodo en la formación del médico, de adquisición de conocimientos y habilidades desde una perspectiva muy diferente a lo aprendido en la universidad. Las actividades se dividen entre la atención a los pacientes y sus familiares y presentación en sesiones clínicas, que pueden conducir a sobrecarga emocional y física ${ }^{(1)}$.

El síndrome de Burnout (SB) es un estado de agotamiento emocional y cognitivo que permea a todas las especialidades médicas y puede influir en la calidad de la formación en la especialidad. En un estudio realizado con residentes de Pediatría, a través de grupos focales, el SB se asoció a auto reporte de actitudes y conductas inadecuadas hacia el paciente. Se ha reportado además el impacto del desgaste en residentes de Pediatría, sobre la calidad de la atención ${ }^{(2,3)}$. Algunos programas de residencia, han tomado en consideración, medidas preventivas del agotamiento emocional y cognitivo de los residentes, como la reducción de las horas laborales ${ }^{(4)}$. Otros implementaron talleres de interacción entre pares, como un método para hacer frente a la angustia o eventos adversos, para lograr una respuesta adaptativa saludable ${ }^{(5)}$.

La escala más utilizada para la búsqueda y medición del SB es la de Maslach Burnout Inventory (MBI). Tiene 3 subescalas: agotamiento emocional (AE), que consiste en la disminución de recursos emocionales y personales del profesional; la Despersonalización (DP), en el que la falta de recursos emocionales lleva al profesional a ver al usuario como un objeto y el tercer componente es la realización personal (RP), que actúa como un factor protector ${ }^{(6)}$.

En los residentes médicos existe tendencia a conductas sedentarias, la cual sumado al estrés aumenta el riesgo cardiovascular ${ }^{(7)}$. La actividad física beneficia el estado de salud debido a las diversas adaptaciones cardiovasculares ${ }^{(8)}$. Sin embargo, son escasos los estudios que lo asocian al SB.

El objetivo del presente estudio fue evaluar el efecto de una actividad física (clases de Zumba) sobre el Síndrome de Burnout en un grupo de residentes de pediatría de un hospital Pediátrico universitario de referencia.

\section{MATERIALES Y MÉTODOS}

Se realizó un estudio de intervención no aleatorizado controlado abierto.

\section{Población e Intervención}

Fueron incluidos residentes de Pediatría de un hospital universitario pediátrico de referencia, en el periodo comprendido entre noviembre del 2016 a enero del 2017. Previo consentimiento informado, fueron asignados a recibir 2 horas semanales de clases de zumba por 10 semanas (grupo estudio) o ninguna intervención (grupo control). El punto de corte principal fue la presencia del SB.

La Zumba: es un ejercicio aeróbico al ritmo de música latina (merengue, samba, reggaetón, cumbia y salsa).

\section{Instrumento de evaluación}

Para determinar la presencia del SB, se han utilizado los resultados de las 3 dimensiones del cuestionario de Maslach (MBI): agotamiento emocional, despersonalización y realización personal, en su versión en español con sus puntuaciones respectivas, El diagnóstico del SB se estableció con puntaje $>26$ en la dimensión $\mathrm{AE}$, $>9$ en la $\mathrm{DP} \mathrm{y}<34$ para $\mathrm{RP}$.

\section{Reclutamiento}

Tuvo 3 etapas. En la primera, se suministró el MBI a todos los residentes. En la segunda se procedió a la asignación de grupos de acuerdo a la posibilidad de poder asistir a las clases de Zumba por 10 semanas. En la tercera (semana 11) se realizó nuevamente MBI a todos los participantes. En el momento del reclutamiento ningún residente presentaba signos, síntomas o antecedentes de depresión u otro trastorno de conducta o personalidad. Otras variables fueron: edad, género, convivencia, actividades sociales, físicas habituales y años de residencia. En la Figura 1 se encuentra el diagrama de Flujo de los participantes.

\section{Tamaño de la muestra}

Para el cálculo del tamaño de la muestra, se consideró un estudio realizado en México, 
utilizando la prueba $\mathrm{T}$, con una magnitud del efecto de 0,73; desviación típica de 0,48 y magnitud del efecto normalizado de 1,52; se necesitó incluir 21participantes en cada grupo, con un error alfa de 0,05 y una potencia de $80 \%$.

\section{Análisis de los datos}

Los datos fueron analizados en el SPSSv21. Se utilizaron medias con desvío estándar y porcentajes. Para la asociación de las variables se utilizó la prueba de Chi cuadrado de Pearson, el test exacto de Fisher y la T Student. Se consideró un error alfa inferior al 5\%.

Aspectos éticos El protocolo de estudio fue aprobado por el comité de ética de la institución con consentimiento informado.

\section{RESULTADOS}

Fueron incluidos 47 residentes, 23 en el grupo estudio y 24 en el grupo control. Los datos demográficos, composición familiar, actividades físicas y años de residencia en ambos grupos se encuentran en la Tabla 1. Los resultados del cuestionario MBI Pre intervención y los principales componentes del diagnóstico del SB se muestra en la Tabla 2. Los datos post intervención, muestran las principales modificaciones de los componentes del $\mathrm{SB}$, especialmente el agotamiento emocional que se redujo en un $47,8 \%$ y la baja realización personal en un $21,3 \%$. Tabla 3 .

Aunque el porcentaje de SB fue mayor después de 10 semanas en el grupo control $37,5 \%$ vs 45,4 . $p=0,76$, la diferencia no fue significativa.

El beneficio absoluto del ejercicio fue de $34,8 \%$ (IC 95\% 9-60) y el NNT es de 2,8.

Tabla 1. Características demográficas, actividad física y años de residencia en pediatría de los participantes del estudio $\mathrm{N}=47$.

\begin{tabular}{|c|c|c|c|}
\hline & $\begin{array}{c}\text { Grupo estudio } \\
n=23\end{array}$ & $\begin{array}{l}\text { Grupo control } \\
n=24\end{array}$ & $\mathbf{P}$ \\
\hline Edad & $26,5 \pm 1,7$ & $28 \pm 2,1$ & $0,01^{1}$ \\
\hline \multicolumn{4}{|l|}{ Media DE } \\
\hline & N (\%) & $N(\%)$ & \\
\hline Sexo femenino & $22(95,6)$ & $18(75)$ & $0,03^{2}$ \\
\hline \multicolumn{4}{|l|}{ Estructura familiar } \\
\hline Vive solo & $7(30,4)$ & $9(37,4)$ & $\mathrm{NS}^{2}$ \\
\hline Practica deporte & $6(26)$ & $4(16,6)$ & $\mathrm{NS}^{3}$ \\
\hline Asiste a gimnasio & $3(13)$ & $3(12,5)$ & $\mathrm{NS}^{3}$ \\
\hline \multicolumn{4}{|l|}{ Año de residencia } \\
\hline R1 & $6(26)$ & $10(41,6)$ & $\mathrm{NS}^{2}$ \\
\hline R2 & $10(43,4)$ & $6(25)$ & $\mathrm{NS}^{2}$ \\
\hline R3 & $5(21,7)$ & $10(41,6)$ & $\mathrm{NS}^{2}$ \\
\hline
\end{tabular}


otro metaanálisis que incluyó sólo estudios clínicos, concluyó que las intervenciones para reducir el SB en médicos producen beneficios pequeños, si no van acompañados de programas de residencia y sistemas de salud orientados a promover el bienestar, ambiente saludable de trabajo y promoción de valores como la compasión y tolerancia ${ }^{(13)}$. Este enfoque de prevención e intervenciones holísticas, se han reflejado en numerosos estudios, tanto cualitativos como cuantitativos, realizados desde la perspectiva de los médicos residentes y de las instituciones de salud ${ }^{(5)}$. El ambiente de trabajo hostil, maltrato físico y verbal, discriminación de género, acoso, el abuso e intento de suicidio se han relacionado con SB de acuerdo a los resultados de una encuesta nacional a residentes de todas las especialidades médicas, realizada en EEUU ${ }^{(16)}$. Otras intervenciones que han tenido resultado positivo se refieren a las prácticas de Mindfulness y las promociones de las capacidades de resiliencia y del entorno saludable del aprendizaje de la especialidad $^{(17,18)}$.

El presente estudio tiene varias limitaciones. Los grupos no fueron comparables en edad y en sexo, porque la mayoría del plantel de residentes son mujeres. Los residentes que se encontraban haciendo

\section{REFERENCIAS}

1. Athié Gutierrez C, Cardiel EL, Camacho J, Avellaneda VM, Terrones A, Cabrera N, et al. Burnout en médicos internos de pregrado del Hospital General de México Dr . Eduardo Liceaga. Inv Ed Med. 2016;5(18):102-7.

2. Baer TE, Feraco AM, Tuysuzoglu Sagalowsky S, Williams D, Litman HJ, Vinci RJ. Pediatric Resident Burnout and Attitudes Toward Patients. Pediatrics. 2017;139(3):e20162163. doi: http://dx.doi.org/10.1542/ peds.2016-2163

3. Brunsberg KA, Landrigan CP, Garcia BM, Petty CR, Sectish TC, Simpkin AL, et al. Association of Pediatric Resident Physician Depression and Burnout With Harmful Medical Errors on Inpatient Services. Acad Med. 2019;94(8):1150-1156. doi: http://dx.doi.org/10.1097/ACM. 0000000000002778 pasantías fuera del hospital eran varones y no pudieron participar de la intervención. El escaso número de participantes no permitió analizar el efecto de otros factores individuales como la composición familiar. Tampoco hemos analizado el ambiente laboral en general y el entorno de aprendizaje. Una de las actividades del hospital donde se realizó el estudio, es el día del atuendo divertido, en el que los residentes y pacientes visten con trajes de personajes infantiles y así realizan la atención de los niños, en un entorno musical. Además el coro de residentes del hospital que se renueva cada año, se presentan ante los pacientes y familiares en fechas especiales. Sin embargo resultados más concluyentes sobre el impacto de una actividad física grupal sobre el SB se podrán obtener con su implementación con un mayor tamaño muestral y teniendo en consideración las variabilidades individuales como la personalidad de cada uno, resiliencia y la práctica del mindfulness.

\section{CONCLUSIONES}

La introducción de clases de Zumba 2 veces por semana, redujo el Síndrome de Burnout en residentes de pediatría de un hospital universitario, en un $35 \%$.

4. Busireddy KR, Miller JA, Ellison K, Ren V, Qayyum R, Panda M. Efficacy of Interventions to Reduce Resident Physician Burnout: A Systematic Review. J Grad Med Educ. 2017;9(3):294-301. doi: http://dx.doi.org/10.4300/J GME-D-16-00372.1

5. Govindan M, Keefer P, Sturza J, Stephens MR, Malas N. Empowering Residents to Process Distressing Events: A Debriefing Workshop. MedEdPORTAL. 2019;15:10809. doi:http://dx.doi.org/10.15766/mep_2374-8265.10809

6. Maslach C, Jackson SE, Leiter MP. The Maslach Burnout Inventory Manual. In: Zalaquett $\mathrm{CP}$, Wood RJ, editors. Evaluating Stress: a book of resources [Internet]. 1997[citado May 2016]; p. 191-217. Available from: https://www.researchgate.net/publication/277816643 
Tabla 2. El síndrome de Burnout y sus componentes en residentes de pediatría pre- intervención con clases de Zumba N=47.

\begin{tabular}{lccc}
\hline & $\begin{array}{c}\text { Grupo estudio } \\
\mathbf{n = 2 3}\end{array}$ & $\begin{array}{c}\text { Grupo control } \\
\mathbf{n = 2 4}\end{array}$ & $\mathbf{P}$ \\
\hline & $\mathbf{N}(\%)$ & $\mathbf{N}(\%)$ & \\
S. Burnout & $12(52,2)$ & $9(37,5)$ & $\mathrm{NS}^{1}$ \\
AE nivel Alto & $17(73,9)$ & $17(70,8)$ & $\mathrm{NS}^{1}$ \\
DP nivel Alto & $17(73,9)$ & $18(75)$ & $\mathrm{NS}^{1}$ \\
RP nivel bajo & $15(51,7)$ & $14(48,3)$ & $\mathrm{NS}^{1}$ \\
\hline $1=\mathrm{r} 2$ & &
\end{tabular}

Tabla 3. El síndrome de Burnout y sus componentes en residentes de pediatría post- intervención con clases de Zumba $\mathrm{N}=47$.

\begin{tabular}{lcccc}
\hline & $\begin{array}{c}\text { Grupo estudio } \\
\mathbf{n = 2 3}\end{array}$ & $\begin{array}{c}\text { Grupo control } \\
\mathbf{n = 2 4}\end{array}$ & $\begin{array}{c}\text { OR } \\
(\mathbf{I c 9 5 \% )}\end{array}$ & $\mathbf{P}$ \\
\hline S. Burnout & $\mathbf{N}(\mathbf{\%})$ & $\mathbf{N}(\mathbf{\%})$ & & \\
AE nivel Alto & $11(47,4)$ & $11(45,8)$ & $0,25(0,65-0,9)$ & 0,03 \\
DP nivel Alto & $18(78,3)$ & $17(70,8)$ & $0,37(0,1-1,2)$ & $\mathrm{NS}^{2}$ \\
RP nivel bajo & $7(30,4)$ & $18(75)$ & $1,2(0,30-4,6)$ & $\mathrm{NS}^{2}$ \\
\hline
\end{tabular}

${ }^{1=}$ Test Exacto de Fisher

${ }^{2}=\mathrm{X} 2$

\section{DISCUSIÓN}

La prevalencia del SB encontrada en el presente estudio previa intervención, fue muy elevada, aunque inferior a la reportada por Medina y cols. quienes evaluaron la presencia del SB en 18 residentes de Pediatría en la ciudad de Resistencia, Argentina, y la totalidad presentaron SB. De acuerdo a un metaanálisis la prevalencia general del Burnout en residentes de todas las especialidades médicas, fue de $37,5 \%$ y en los residentes de pediatría en particular fue del $30 \%{ }^{(9)}$. Puranitee y cols, no encontraron Burnout en un grupo de 51 residentes de Pediatría en Tailandia, mientras que en Arabia Saudita, Hameed y cols, encontraron $81 \%$ de casos de Burnout en 181 residentes de todas las especialidades $^{(10,11)}$.

La mayoría de los estudios demuestran que los niveles de Burnout en médicos alcanzan cifras cercanas a una epidemia ${ }^{(12)}$. Sin embargo, no hay muchos estudios sobre intervenciones que ayuden a mitigarlo. En el presente estudio la implementación de las clases de Zumba tuvo un impacto positivo en la disminución del porcentaje de SB. Es posible que haya otros factores, no medidos en este estudio, que no puedan ser modificados solo por la actividad física, tales como la personalidad, la manera de enfrentar situaciones adversas y la resiliencia ${ }^{(13)}$. El aumento del porcentaje del SB en el grupo control después de 10 semanas, se atribuyó al azar, considerando que la diferencia entre los porcentajes no fue estadísticamente significativa. Méndez Cerezo y cols. compararon los niveles de $\mathrm{SB}$ en dos grupos de residentes $(\mathrm{N}=20)$, luego de una intervención con actividad física aeróbica durante un periodo de 8 semanas. Observaron una reducción del 35\% del SB en el grupo intervención, resultado muy similar al encontrado en el presente estudio ${ }^{(14)}$. La revisión sistemática y metaanálisis, tanto, de estudios clínicos como observacionales, que evaluaron los resultados de los programas de intervención ya sea en la prevención o en el manejo del SB en médicos, mostró una reducción global del 54 al $44 \%^{(15)}$. Sin embargo, 
7. Lamar DL, Chou SHS, Medverd JR, Swanson JO. Sedentary Behavior in the Workplace: A Potential Occupational Hazard for Radiologists. Curr Probl Diagn Radiol. 2016;45(4):253-7. doi: http://dx.doi.org/10.1067/ j.cpradiol.2015.10.007

8. Warburton DER. Health Benefits of Physical Activity?: a Strengths-Based Approach. J Clin Med. 2019;8:2044. doi: http://dx.doi.org/10.3390/jcm8122044

9. Rodrigues H, Cobucci R, Oliveira A, Cabral JV, Medeiros L, Gurgel K, et al. Burnout syndrome among medical residents: A systematic review and meta-analysis. PLoS One. 2018;13(11):e0206840. doi: http://dx.doi.org/ 10.1371/journal.pone.0206840

10. Puranitee P, Stevens FFCJ, Pakakasama S, Plitponkarnpim A, Vallibhakara SA, Busari JO, et al. Exploring burnout and the association with the educational climate in pediatric residents in Thailand. BMS Med Educ. 2019;245. doi: https://doi.org/10.1186/s12909$019-1687-7$

11. Hameed TK, Masuadi E, Al Asmary NA, Al-Anzi FG, Al Dubayee MS. A study of resident duty hours and burnout in a sample of Saudi residents. BMC Med Educ. 2018;18(1):1-6. doi: http://dx.doi.org/10.1186/s12909-018$1300-5$

12. Panagioti M, Geraghty K, Johnson J, Zhou A, Panagopoulou E, Chew-Graham C, et al. Association between Physician Burnout and Patient Safety, Professionalism, and Patient Satisfaction: a systematic review and meta-analysis. JAMA Intern Med. 2018;178(10) :1317-30. http://dx.doi.org/10.1001/jamainternmed.2018.3713
13. Panagioti M, Panagopoulou E, Bower P, Lewith G, Kontopantelis E, Chew-Graham C, et al. Controlled interventions to reduce burnout in physicians: a systematic review and meta-analysis. JAMA Intern Med. 2017;177(2):195-205. doi: 10.1001/jamainternmed.2016.7674

14. Méndez-Cerezo Á. Síndrome de Burnout en médicos residentes. Ejercicio como estrategia para su disminución. An Médicos México [Internet]. 2011[citado May 2016];56(2):79-84. Available from: https://www.medigra phic.com/pdfs/abc/bc-2011/bc112d.pdf

15. West CP, Dyrbye LN, Erwin PJ, Shanafelt TD. Interventions to prevent and reduce physician burnout?: a systematic review and meta-analysis. Lancet. 2016;6736(16). doi: http://dx.doi.org/10.1016/S01406736(16)31279-X

16. Hu YY, Ellis RJ, Hewitt DB, Yang AD, Cheung EO, Moskowitz JT, et al. Discrimination, abuse, harassment, and burnout in surgical residency training. N Engl J Med. 2019;381(18):1741-52. doi: http://dx.doi.org/10.1056/ NEJMsa1903759

17. Olson K, Kemper KJ, Mahan JD. What factors promote resilience and protect against burnout in first-year pediatric and medicine-pediatric residents? J Evid Based Complementary Altern Med. 2015;20(3):192-8. doi: http://dx.doi.org/10.1177/2156587214568894

18. Olson K, Kemper KJ, Mahan JD. What factors promote resilience and protect against burnout in first-year pediatric and medicine-pediatric residents? J Evid Based Complementary Altern Med. 2015;20(3):192-8. doi: http://dx.doi.org/10.1177/2156587214568894 